\title{
ALTERAÇÕES NOS PARÂMETROS PRÉ E PÓS-OPERATÓRIOS DE PACIENTES COM SÍNDROME METABÓLICA, SUBMETIDOS A BYPASS GASTROINTESTINAL EM Y DE ROUX
}

\section{Changes in the pre- and postoperative parameters in metabolic syndrome patients submitted to roux-en-y gastric bypass}

\author{
Paulo Afonso Nunes NASSIF ${ }^{1}$, Andre Donato LOPES ${ }^{2}$, Gabriel Lima LOPES ${ }^{2}$, \\ Priscila Reis MARTINS ${ }^{2}$, Lucas Eduardo PEDRI ${ }^{2}$, Michelle VARASCHIM ${ }^{1}$, Denise Serpa BOPP ${ }^{1}$
}

ABCDDV/664

Nassif PAN, Lopes AD, Lopes GL, Martins PR, Pedri LE, Varaschim M, Bopp DS. Alterações nos parâmetros pré e pós-operatórios de pacientes com síndrome metabólica, submetidos a bypass gastrointestinal em y de roux. ABCD Arq Bras Cir Dig 2009;22(3):165-70

RESUMO - Racional - A epidemia mundial de sobrepeso e obesidade afeta aproximadamente 1,7 bilhão de pessoas, havendo aumento nos casos de obesidade mórbida de $80 \%$ para mulheres e de $400 \%$ para homens desde 1970 . A síndrome metabólica é transtorno complexo relacionado com resistência à insulina e obesidade abdominal, provocando alterações no metabolismo da glicose em vários tecidos e hiperinsulinemia. Objetivo - Verificar as alterações nos parâmetros pré e pós-operatórios em pacientes obesos mórbidos com síndrome metabólica, submetidos a bypass gastrointestinal em Y de Roux. Método - Casuística de 74 pacientes obesos, que apresentavam síndrome metabólica e submetidos a procedimento cirúrgico. Foram avaliados, além do IMC, a relação cintura-quadril, pressão arterial, colesterol total, HDL, LDL, triglicerídeos, glicemia de jejum no pré-operatório e no $1^{\circ}$ e $4^{\circ}$ meses de pós-operatório. Para variáveis que apresentaram simetria na sua distribuição, foi usado o teste t de Student para amostras pareadas. Para variáveis com distribuição assimétrica, foi usado o teste não-paramétrico de Wilcoxon. Valores de $\mathrm{P}<0,05$ indicaram significância estatística. Resultados - Obteve-se redução no $1^{\circ}$ mês do pós-operatório no peso de 7,96\%, IMC de $8 \%$, relação cintura/quadril de $1,35 \%$, colesterol total de $17,95 \%$, HDL de $14,5 \%$, LDL de $15,31 \%$, TAG's de $30,29 \%$ e glicemia de $9,75 \%$; no $4^{\circ}$ mês, observou-se redução no peso de $14,94 \%$, IMC de 20,08\%, relação cintura/quadril de 5,96\%, colesterol total de 21,27\%, HDL de 3,53\%, LDL de 23,72\%, TAG's de 29,6\% e na glicemia de 10,34\%. Conclusãa - Os parâmetros avaliados neste estudo tiveram melhora estatisticamente significante, com exceção do HDL-colesterol.

DESCRITORES - Síndrome metabólica. Obesidade. Bypass gastrointestinal em Y de Roux.

\section{INTRODUÇÃO}

A síndrome metabólica é transtorno complexo, caracterizada por conjunto de fatores de risco cardiovasculares, relacionados com resistência à insulina e obesidade abdominal. A obesidade provoca alterações no metabolismo da glicose em vários tecidos, o que promove hiperinsulinemia - apesar de também haver outros mecanismos reduzidos na reciclagem hepática de insulina. Atualmente, estima-se que $24 \%$ da população adulta dos EUA e entre 50 e $60 \%$ na população acima de 50 anos possuem essa síndrome e algumas projeções indicam que, em 2010, os EUA terão de 50 a 75 milhões de pessoas afetadas ${ }^{3,4,14,16}$.

Por proposta feita pela Organização Mundial de Saúde, define-se síndrome metabólica quando o indivíduo possuir dois ou mais dos seguintes componentes 5,7:1) glicemia de jejum $>125 \mathrm{mg} / \mathrm{dL}$, glicemia aleatória $>200 \mathrm{mg} / \mathrm{dL}$

Trabalho realizado no ${ }^{1}$ Serviço de Cirurgia do Hospital Nossa Senhora do Rocio, Campo Largo, PR, Brasil. ${ }^{2}$ Curso de Medicina da Faculdade Evangélica do Paraná, Curitiba, PR, Brasil.

Endereço para correspondência: Paulo Afonso Nunes Nassif, e-mail: paulonassif@terra.com.br ou com um TOTG $>200 \mathrm{mg} / \mathrm{dL}$ ou $140-200 \mathrm{mg} / \mathrm{dL}$ com sintomas clássicos de hiperglicemia; 2) pressão arterial elevada $(>130 />85 \mathrm{mmHg}) ; 3)$ triglicerídeos plasmáticos elevados (>1,7 mmol $-150 \mathrm{mg} / \mathrm{dL})$; 4) colesterol HDL baixo ( $<40$ para homens e $<50$ para mulheres); 5 ) obesidade central (relação cintura/quadril $>0,9$ para homens e $>0,8$ para mulheres) e/ou índice de massa corpórea (IMC) acima de $30 \mathrm{Kg} / \mathrm{m}^{2} ; 6$ ) microalbuminúria $>15 \mu \mathrm{g} / \mathrm{min}$ ou relação albumina/creatinina urinária $>30 \mathrm{mg}$.

Outros componentes estudados nesta síndrome também foram relacionados com a resistência à insulina e/ou tendem a agrupar-se com a síndrome principal, entre eles: hiperuricemia, proporção aumentada de LDL pequena e densa, aumento da concentração de lipoproteínas remanescentes, distúrbios da coagulação e da fibrinólise (elevação do fibrinogênio), disfunção endotelial, inflamação da parede arterial, angina microvascular e síndrome de ovários policísticos ${ }^{14,18}$

Outros grupos de especialistas apresentaram outras versões para a definição da síndrome (três itens ou mais), como o Programa Nacional de Educação em Colesterol 
(NCEP - EUA, baseado nos fatores de risco para a síndrome) e o Grupo Latino-Americano da Oficina Internacional de Informação em Lípides sendo a obesidade combinada com um fator de risco cardiovascular ${ }^{4,17,18}$.

Nos pacientes obesos mórbidos, os tratamentos conservadores têm se mostrado eficazes em promover melhora da síndrome metabólica; no entanto, os resultados de médio e longo prazos são desapontadores, pois não há manutenção da perda de peso na quase totalidade dos indivíduos. A cirurgia bariátrica tem se mostrado eficaz em promover perda significativa e sustentada de peso em obesos mórbidos. A partir de 1991, várias sociedades médicas internacionais estabeleceram como critério de recomendação da cirurgia bariátrica o insucesso do tratamento clínico em pacientes com IMC $>40$ ou IMC $>35$ nos casos de comorbidades graves associadas, passíveis de reversão com o emagrecimento induzido pela cirurgia9.

Seriam então três os principais motivos pelos quais um procedimento cirúrgico produziria perda de peso: o efeito restritivo de pequena bolsa gástrica, gerando sensação de saciedade precocemente; absorção inadequada de nutrientes provocada pela exclusão de parte do tubo digestivo do trânsito alimentar; e, em terceiro lugar, o trânsito rápido do alimento até as porções mais distais do intestino delgado ${ }^{11,12}$.

A operação de Fobi-Capella é hoje a mais utilizada para o tratamento da obesidade, devido à sua elevada eficiência e baixa morbi-mortalidade ${ }^{7}$.

Estudos recentes relatam a provável relação entre tratamento da síndrome metabólica e a cirurgia bariátrica. Após curto período da realização do procedimento, já se pode observar a normalização da glicemia em $30 \%$ dos pacientes, dos índices lipidêmicos em $70 \%$, da pressão arterial em $61,7 \%$, do estado de hipogonadismo hipogonadotrófico, dos diversos marcadores de hipercoagulabilidade e hipofibrinólise; esses resultados são confirmados pela positiva correlação entre os parâmetros da síndrome e o IMC. A própria redução no peso corporal normaliza os níveis de insulina, possivelmente devido à melhora nos mecanismos sinalizadores da insulina, captação e oxidação de glicose, e redução da oxidação lipídica em relação à oxidação de carboidratos ${ }^{9,16}$.

Os nutrientes alcançam o íleo terminal em cinco minutos após a ingestão de bebidas e de alimentos contendo glicose, e isto estimula a secreção de hormônios entéricos pelas células-L, como o peptídeo YY (PYY - liberado em resposta à presença de bile, gorduras, glicose e aminoácidos) e as incretinas (o GLP-1, Glucagon-Like Peptide - 1, e o GIP, Glucose-dependent Insulinotropic Polyptide). Essas substâncias lentificam o esvaziamento do conteúdo gástrico e diminuem o peristalismo, resultando, portanto, em sinais anoréticos fisiológicos. Mais importante, no caso do diabete melito tipo 2, é a sua capacidade (principalmente das incretinas) de estimular o crescimento de células beta do pâncreas, de secreção de insulina e de torná-la mais efetiva ${ }^{11,12,13}$.

Em resumo, o bypass gástrico em Y de Roux atua inicialmente como operação restritiva, levando à saciedade precoce. A exclusão de parte do segmento estômago-duodeno-jejunal, leva a diminuição dos níveis pós-prandiais de grelina, diminuindo ainda mais o apetite. A presença mais precoce do alimento no íleo terminal leva a maior produção de anorexígenos endógenos (PYY e GLP-1). Através dessas vias hormonais obtém-se o importante efeito antidiabetogênico do procedimento cirúrgico, além de promover adequada perda de peso a longo prazo $^{12}$.

Considerando-se que o desvio do trânsito alimentar promovido cirurgicamente tem a capacidade de alterar a síntese de hormônios e a secreção e sensibilidade da insulina, objetivouse verificar as alterações nos parâmetros pré e pós-operatórios em pacientes obesos mórbidos com síndrome metabólica, submetidos à bypass gastrointestinal em $\mathrm{Y}$ de Roux.

\section{MÉTODO}

O estudo foi realizado em 74 pacientes com obesidade, que apresentavam síndrome metabólica, sendo seus dados coletados a partir de prontuários. Não foram utilizados critérios de escolha dos pacientes quanto ao sexo, idade, naturalidade ou raça e sim, apenas os critérios clínicos que indicavam a operação bariátrica em pacientes portadores dessa síndrome. Todos foram submetidos à by pass gastrointestinal em Y de Roux.

Os parâmetros estudados pré-operatórios foram coletados através de ficha de avaliação composta por identificação (nome, idade, sexo), dados antropométricos (altura, peso, IMC), fatores de riscos (ácido úrico, fibrinogênio, angina microvascular, síndrome de ovários policísticos, proteína C reativa ultra-sensível) e os parâmetros para o diagnóstico da síndrome metabólica (relação cintura-quadril, pressão arterial, colesterol total, HDL, LDL, triglicerídeos, glicemia de jejum). Os dados do pós-operatório foram coletados a partir do primeiro e do quarto mês, utilizando a mesma ficha de avaliação usada para coleta de dados pré-operatórios.

Os resultados foram expressos por médias, medianas, valores mínimos, valores máximos e desvios-padrão ou por frequências e percentuais. Considerando-se que as avaliações dos pacientes após um mês e quatro meses não estavam disponíveis para todos os pacientes, os resultados no préoperatório foram comparados separadamente com os obtidos após um mês e quatro meses. Para tanto, para variáveis que apresentaram simetria na sua distribuição, foi usado o teste $t$ de Student para amostras pareadas. Para variáveis com distribuição assimétrica, foi usado o teste não-paramétrico de Wilcoxon. Valores de $\mathrm{P}<0,05$ indicaram significância estatística.

\section{RESULTADOS}

Na distribuição por sexo incluíram-se 13 (17,57\%) homens e $61(82,43 \%)$ de mulheres. Em relação à angina microvascular $59(84,3 \%)$ a possuíam e $11(15,7 \%)$ não.

$\mathrm{Na}$ Tabela 1 são apresentadas as distribuições de frequências para variáveis qualitativas e valores de média, mediana, valor mínimo, valor máximo e desvio-padrão para variáveis quantitativas, considerando-se a avaliação pré-operatória. 
TABELA 1 - Dados antropométricos e laboratoriais pré-operatórios dos 74 pacientes

\begin{tabular}{|c|c|c|c|c|c|c|}
\hline Variável & $\mathbf{n}$ & Média & Mediana & Mínimo & Máximo & $\begin{array}{l}\text { Desvio- } \\
\text { padrão }\end{array}$ \\
\hline Idade & 73 & 35,40 & 3300 & 19,00 & 62,00 & 10,61 \\
\hline Altura & 71 & 1,66 & 1,66 & 1,50 & 1,88 & 0,08 \\
\hline Peso & 71 & 103,93 & 101,80 & 81,50 & 151,00 & 14,88 \\
\hline IMC & 71 & 37,56 & 37,24 & 28,88 & 50,80 & 3,74 \\
\hline Ácido úrico & 72 & 5,45 & 5,55 & 2,53 & 8,90 & 1,57 \\
\hline Fibrinogênio & 35 & 368,01 & 372,90 & 196,00 & 590,00 & 95,38 \\
\hline $\begin{array}{l}\text { Proteína } \mathrm{C} \\
\text { reativa }\end{array}$ & 33 & 1,96 & 0,73 & 0,13 & 12,00 & 3,10 \\
\hline $\begin{array}{l}\text { Relação cintura/ } \\
\text { quadril }\end{array}$ & 54 & 0,95 & 0,94 & 0,76 & 1,19 & 0,09 \\
\hline PAS & 69 & 124,13 & 120,00 & 90,00 & 150,00 & 12,16 \\
\hline PAD & 69 & 81,88 & 80,00 & 60,00 & 100,00 & 8,75 \\
\hline Colesterol total & 73 & 189,22 & 182,00 & 119,00 & 268,00 & 36,60 \\
\hline HDL & 72 & 44,67 & 44,00 & 6,00 & 76,00 & 10,44 \\
\hline LDL & 73 & 112,03 & 107,00 & 38,00 & 179,00 & 30,79 \\
\hline TAG's & 73 & 159,19 & 137,00 & 41,00 & 441,00 & 81,77 \\
\hline Glicemia & 74 & 96,55 & 91,00 & 72,00 & 220,00 & 22,61 \\
\hline PAS & 69 & 124,13 & 120 & 90 & 150 & 12,16 \\
\hline PAD & 69 & 81,88 & 80 & 60 & 100 & 8,75 \\
\hline
\end{tabular}

$\mathrm{IMC}=$ índice de massa corpórea

$\mathrm{PAS}=$ pressão arterial sistólica

$\mathrm{PAD}=$ pressão arterial diastólica

TAG's= triglicerídeos

Na Tabela 2, para cada variável, são apresentadas estatísticas descritivas pré-operatória, da avaliação após um mês e da redução percentual ocorrida entre o período pré-operatório e um mês após a operação.

TABELA 2 - Avaliação pré-operatória, após um mês da operação e redução percentual nas variáveis

\begin{tabular}{|c|c|c|c|c|c|c|c|c|}
\hline Variável & Avaliação & n & Média & Mediana & Mínimo & Máximo & $\begin{array}{l}\text { Desvio- } \\
\text { padrão }\end{array}$ & $\begin{array}{c}\text { Valor de } \\
\mathrm{P}^{*}\end{array}$ \\
\hline \multirow{3}{*}{ Peso } & Pré & 43 & 104,94 & 102,50 & 81,50 & 151,00 & 16,41 & \multirow{3}{*}{$<0,001$} \\
\hline & 1 mês & 43 & 96,32 & 93,80 & 65,00 & 139,00 & 14,64 & \\
\hline & Redução (\%) & 43 & 7,96 & 8,98 & 20,44 & $-22,70$ & 6,60 & \\
\hline \multirow{3}{*}{ IMC } & Pré & 43 & 37,44 & 37,24 & 28,88 & 50,80 & 4,20 & \multirow{3}{*}{$<0,001$} \\
\hline & 1 mês & 43 & 34,36 & 33,40 & 26,70 & 46,50 & 3,94 & \\
\hline & Redução (\%) & 43 & 8,00 & 8,88 & 20,47 & $-22,59$ & 6,66 & \\
\hline \multirow{3}{*}{$\begin{array}{l}\text { Relação } \\
\text { cint/quadril }\end{array}$} & Pré & 22 & 0,95 & 0,94 & 0,84 & 1,11 & 0,08 & \multirow{3}{*}{0,137} \\
\hline & 1 mês & 22 & 0,94 & 0,94 & 0,82 & 1,10 & 0,07 & \\
\hline & Redução (\%) & 22 & 1,35 & 1,03 & 10,29 & $-6,74$ & 4,34 & \\
\hline \multirow{3}{*}{$\begin{array}{l}\text { Colesterol } \\
\text { total }\end{array}$} & Pré & 38 & 190,86 & 187,50 & 136,00 & 268,00 & 35,35 & \multirow{3}{*}{$<0,001$} \\
\hline & 1 mês & 38 & 155,37 & 145,50 & 92,00 & 264,00 & 33,40 & \\
\hline & Redução (\%) & 38 & 17,95 & 18,21 & 45,67 & $-30,05$ & 13,28 & \\
\hline \multirow{3}{*}{ HDL } & Pré & 17 & 44,22 & 42,00 & 32,00 & 65,80 & 8,92 & \multirow{3}{*}{$0,005^{* *}$} \\
\hline & 1 mês & 17 & 37,35 & 34,00 & 23,00 & 106,00 & 18,36 & \\
\hline & Redução (\%) & 17 & 14,05 & 21,88 & 51,37 & $-135,56$ & 40,66 & \\
\hline \multirow{3}{*}{ LDL } & Pré & 17 & 118,75 & 113,00 & 71,00 & 174,00 & 31,50 & \multirow{3}{*}{0,016} \\
\hline & 1 mês & 17 & 100,40 & 87,00 & 28,00 & 189,00 & 36,27 & \\
\hline & Redução (\%) & 17 & 15,31 & 17,14 & 61,64 & $-41,04$ & 22,62 & \\
\hline \multirow{3}{*}{ TAG's } & Pré & 17 & 207,32 & 188,00 & 102,00 & 425,00 & 94,43 & \multirow{3}{*}{0,002} \\
\hline & 1 mês & 17 & 126,00 & 116,00 & 35,00 & 215,00 & 51,28 & \\
\hline & Redução (\%) & 17 & 30,29 & 40,85 & 88,05 & $-110,78$ & 41,86 & \\
\hline \multirow{3}{*}{ Glicemia } & Pré & 39 & 100,75 & 93,00 & 73,00 & 220,00 & 26,05 & \multirow{3}{*}{$<0,001$} \\
\hline & 1 mês & 39 & 89,28 & 85,00 & 68,00 & 193,00 & 20,64 & \\
\hline & Redução (\%) & 39 & 9,75 & 8,60 & 41,96 & $-21,67$ & 13,64 & \\
\hline \multirow{3}{*}{ PAS } & Pré & 41 & 124,76 & 120 & 90 & 150 & 13,78 & \multirow{3}{*}{$<0,001$} \\
\hline & 1 mês & 41 & 115,37 & 110 & 90 & 150 & 10,98 & \\
\hline & Redução (\%) & 41 & 6,84 & 7,14 & 28,57 & 9,09 & 9,86 & \\
\hline \multirow{3}{*}{ PAD } & Pré & 41 & 81,59 & 80 & 60 & 100 & 8,83 & \multirow{3}{*}{$<0,001$} \\
\hline & 1 mês & 41 & 75,37 & 70 & 60 & 100 & 8,4 & \\
\hline & Redução (\%) & 41 & 7,03 & 5,88 & 33,33 & 14,29 & 10,71 & \\
\hline
\end{tabular}

* Teste t de Student para amostras pareadas, $\mathrm{P}<0,05$

** Teste não-paramétrico de Wilcoxon, $\mathrm{P}<0,05$

$\mathrm{IMC}=$ índice de massa corpórea

PAS $=$ pressão arterial sistólica

$\mathrm{PAD}=$ pressão arterial diastólica

TAG's= triglicerídeos
Na Tabela 3, para cada variável, são apresentadas estatísticas descritivas da avaliação pré-operatória, da avaliação após quatro meses e da redução percentual ocorrida entre o período pré-operatório e quatro meses após a operação.

TABELA 3 - Avaliação pré-operatória, após quatro meses da operação e redução percentual nas variáveis

\begin{tabular}{|c|c|c|c|c|c|c|c|c|}
\hline Variável & Avaliação & $\mathbf{n}$ & Média & Mediana & Mínimo & Máximo & $\begin{array}{l}\text { Desvio } \\
\text { padrão }\end{array}$ & $\begin{array}{c}\text { Valor de } \\
P^{*}\end{array}$ \\
\hline \multirow{3}{*}{ Peso } & Pré & 22 & 106,31 & 104,65 & 81,50 & 140,00 & 16,63 & \multirow{3}{*}{$<0,001$} \\
\hline & 4 meses & 22 & 84,95 & 83,20 & 64,70 & 114,60 & 13,66 & \\
\hline & Redução (\%) & 22 & 19,94 & 20,47 & 26,31 & $-2,33$ & 5,98 & \\
\hline \multirow{3}{*}{ IMC } & Pré & 22 & 36,69 & 36,33 & 28,88 & 44,95 & 3,82 & \multirow{3}{*}{$<0,001$} \\
\hline & 4 meses & 22 & 29,25 & 28,25 & 24,10 & 38,20 & 3,24 & \\
\hline & Redução (\%) & 22 & 20,08 & 20,45 & 28,31 & $-3,55$ & 6,38 & \\
\hline \multirow{3}{*}{$\begin{array}{l}\text { Relação } \\
\text { cint/quadril }\end{array}$} & Pré & 13 & 1,00 & 1,00 & 0,84 & 1,19 & 0,11 & \multirow{3}{*}{0,020} \\
\hline & 4 meses & 13 & 0,93 & 0,93 & 0,83 & 1,10 & 0,07 & \\
\hline & Redução (\%) & 13 & 5,96 & 7,50 & 17,61 & $-5,77$ & 8,12 & \\
\hline \multirow{3}{*}{$\begin{array}{l}\text { Colesterol } \\
\text { total }\end{array}$} & Pré & 22 & 202,61 & 191,00 & 140,00 & 268,00 & 37,91 & \multirow{3}{*}{$<\mathbf{0 , 0 0 1}$} \\
\hline & 4 meses & 22 & 157,95 & 153,50 & 119,00 & 218,80 & 28,83 & \\
\hline & Redução (\%) & 22 & 21,27 & 21,98 & 40,47 & $-4,29$ & 10,69 & \\
\hline \multirow{3}{*}{ HDL } & Pré & 14 & 45,14 & 43,50 & 35,00 & 58,90 & 8,83 & \multirow{3}{*}{$0,126^{* *}$} \\
\hline & 4 meses & 14 & 42,65 & 41,00 & 30,00 & 72,00 & 10,61 & \\
\hline & Redução (\%) & 14 & 3,53 & 8,47 & 33,62 & $-75,61$ & 26,30 & \\
\hline \multirow{3}{*}{ LDL } & Pré & 13 & 120,45 & 107,00 & 62,90 & 179,00 & 36,02 & \multirow{3}{*}{0,001} \\
\hline & 4 meses & 13 & 90,07 & 89,00 & 43,90 & 140,00 & 25,26 & \\
\hline & Redução (\%) & 13 & 23,72 & 24,75 & 48,85 & $-6,00$ & 14,90 & \\
\hline \multirow{3}{*}{ TAG's } & Pré & 13 & 200,92 & 211,00 & 72,00 & 425,00 & 91,88 & \multirow{3}{*}{0,007} \\
\hline & 4 meses & 13 & 117,77 & 105,00 & 58,00 & 189,00 & 47,22 & \\
\hline & Redução (\%) & 13 & 29,60 & 46,97 & 73,45 & $-62,50$ & 44,53 & \\
\hline \multirow{3}{*}{ Glicemia } & Pré & 20 & 100,80 & 95,00 & 74,00 & 220,00 & 29,99 & \multirow{3}{*}{$0,002^{* *}$} \\
\hline & 4 meses & 20 & 87,40 & 86,50 & 73,00 & 124,00 & 10,50 & \\
\hline & Redução (\%) & 20 & 10,34 & 10,33 & 43,64 & $-21,62$ & 13,87 & \\
\hline \multirow{3}{*}{ PAS } & Pré & 20 & 123,50 & 120 & 110 & 150 & 13,09 & \multirow{3}{*}{0,107} \\
\hline & 4 meses & 20 & 119,00 & 120 & 110 & 160 & 12,52 & \\
\hline & Redução (\%) & 20 & 3,18 & 0 & 21,43 & 9,09 & 9,20 & \\
\hline \multirow{3}{*}{ PAD } & Pré & 20 & 81,00 & 80 & 70 & 100 & 9,54 & \multirow{3}{*}{0,074} \\
\hline & 4 meses & 20 & 76,75 & 80 & 60 & 105 & 10,55 & \\
\hline & Redução (\%) & 20 & 4,68 & 0 & 25 & 14,29 & 12,34 & \\
\hline
\end{tabular}

* Teste $t$ de Student para amostras pareadas, $\mathrm{P}<0,05$

** Teste não-paramétrico de Wilcoxon, $\mathrm{P}<0,05$

IMC = índice de massa corpórea

PAS $=$ pressão arterial sistólica

$\mathrm{PAD}=$ pressão arterial diastólica

TAG's= triglicerídeos

\section{DISCUSSÃO}

A redução de 5 a $10 \%$ do peso corporal após a operação gástrica tem sido apontada como eficaz em melhorar o controle do diabete ou promover reversão da doença nas suas fases iniciais. No entanto, estes conhecidos dados referem-se aos pacientes com sobrepeso ou obesidade grau I. Nos casos de obesidade grau III e na superobesidade (IMC > 50), esta redução, embora útil, é muito modesta para atingir os objetivos de tratamento do diabete ${ }^{3}$.Mattar et al. ${ }^{14}$ afirmam que $15 \%$ das crianças e adolescentes norte-americanos são obesos e que mais de $20 \%$ dessas faixas etárias já são consideradas de risco; de acordo com as progressões, $40 \%$ da população será obesa no ano 2025 .

Batsis et al. ${ }^{2}$ demonstraram que a reversibilidade da síndrome metabólica depende mais do percentual de perda de peso perdido do que outra característica clínica ou demográfica. Esse estudo relatou que a cirurgia bariátrica reduz os níveis sanguíneos de leptina e grelina, melhora os níveis de adiponectina e a sensibilidade à insulina reduz o turnover de ácidos graxos, o que promove redução do processo inflamatório sistêmico e melhora a função endotelial. 
Ramos et al. ${ }^{16}$ encontraram redução no peso corporal de $11,1 \%$ com um mês e de 19,2\% com três de pós-operatório, enquanto Alamo et al. ${ }^{1}$ encontraram redução de $10,9 \%$ com primeiro mês e de $21,6 \%$ no terceiro. Neste estudo foi observada redução do peso corporal de 7,96\% ( \pm 6,6\%) após um mês e de $19,94 \%$ ( $(5,98 \%)$ após quatro da operação. Isso é benéfico para os pacientes, pois reduz a prevalência da síndrome metabólica, de diabete melito, de dislipidemia, de esteatose hepática e de hipertensão arterial por melhorar a sensibilidade periférica à insulina, reduzir os níveis de glicose e de leptina, diminuindo o custo total de cuidados de saúde, pela menor necessidade de utilizar antidiabéticos orais, insulina e medicamentos anti-hipertensivos.

$\mathrm{O}$ IMC tem sido visto como um importante marcador da condição clínica dos pacientes com a síndrome metabólica em acompanhamentos ambulatoriais. Eberly et al. ${ }^{6}$ descreveram que $45,2 \%$ dos pacientes que apresentam o índice elevado têm a síndrome. Batsis et al. ${ }^{2}$ relataram que no grupo de pacientes submetidos ao tratamento cirúrgico houve redução de $88 \%$ para $37 \%$ nos casos de obesidade, enquanto no grupo não-cirúrgico foi visto apenas queda discreta de $88 \%$ para $75 \%$ (com $\mathrm{P}<0,001$ ), quando o IMC foi usado como critério diagnóstico de obesidade.

Ramos et al. ${ }^{16}$ descreveram como o IMC dos seus 30 pacientes evoluiu com o tratamento cirúrgico. No préoperatório o índice médio era de $48,4 \mathrm{~kg} / \mathrm{m}^{2}$; um mês após reduziu para $43 \mathrm{~kg} / \mathrm{m}^{2}$ e depois de 3 meses para $39,1 \mathrm{~kg} /$ $\mathrm{m}^{2}$. Alamo et al. ${ }^{1}$ também fizeram acompanhamento com 30 pacientes para verificar a evolução no índice de massa corpórea; no pré-operatório, a média do IMC era de 41,2 $\mathrm{kg} / \mathrm{m}^{2}$; um mês após a operação reduziu para $36,6 \mathrm{~kg} / \mathrm{m}^{2}$ e após três para $32,2 \mathrm{~kg} / \mathrm{m}^{2}$. Carvalho et al..$^{4}$, de maneira semelhante a estes autores, obteve média no índice no pré-operatório de $49 \mathrm{~kg} / \mathrm{m}^{2}$; um mês após a operação reduziu para $43,5 \mathrm{~kg} / \mathrm{m}^{2}$ e após 3 três para $40 \mathrm{~kg} / \mathrm{m}^{2}$, quando analisaram 47 mulheres obesas.

Nesta pesquisa pôde-se notar queda no IMC nos dois grupos estudados. No grupo onde o IMC foi analisado um mês após a operação, observou-se diminuição de 37,44 $( \pm 4,2)$ para $34,36( \pm 3,94)$, o que significou redução de aproximadamente $8 \%$; já no grupo onde o IMC foi analisado quatro meses após, observou-se diminuição de 36,69 $( \pm 3,82)$ para $29,25( \pm 3,24)$, o que significou redução de aproximadamente $20,08 \%$.

As operações que mantêm o alimento em contato com o fundo gástrico - sítio de maior produção de grelina -, podem ser menos eficazes em diminuir a produção desse hormônio se comparadas com aquelas que excluem ou promovem a ressecção desse segmento, visto que nesta observa-se intensa redução da ingestão alimentar acompanhada da paradoxal redução do apetite. Entretanto, parece não haver correlação positiva entre controle do diabete e alterações nos níveis de grelina, mesmo utilizando as técnicas de melhor resultado, pois esse hormônio parece dificultar a liberação e a ação da insulina ${ }^{11,12}$.

As técnicas de bypass eliminam o controle do músculo pilórico, alterando o esvaziamento gástrico. Quando o conteúdo estomacal altamente concentrado passa ao duodeno, osmoreceptores duodenais causam contração do músculo pilórico, o que previne esvaziamento gástrico tardio, até que se diminua a concentração da bile e do suco pancreático. Qualquer operação que elimine o controle pilórico do conteúdo intestinal resultará em estimulação do peristalismo intestinal e em fluxo do conteúdo para o íleo terminal, com o consequente estímulo da secreção do GLP-1 para a circulação. Operações que estimulam a síntese de GLP-1 têm melhor índice de cura e prevenção do diabete do que operações que somente diminuem a ingestão calórica e a perda de peso, confirmado pelos níveis séricos muito altos desse hormônio até 20 anos após o bypass ${ }^{1,13}$.

Até mesmo perda de peso razoável é suficiente para normalizar a glicemia em $30 \%$ dos casos. Utilizando a técnica de bypass gastrointestinal em Y de Roux, há melhora nos índices de glicose sérica de $91 \%$ e desaparecimento do diabete em $84 \%$. Entretanto, apesar de haver queda significativa da glicemia logo após a operação, não se observa diminuição importante entre o $1^{\circ}$ e o $3^{\circ}$ mês de pós-operatório ${ }^{7,9}$. De acordo com Carvalho et al. ${ }^{4}$, a glicemia foi reduzida de $120 \mathrm{mg} / \mathrm{dL}$ para $105 \mathrm{mg} / \mathrm{dL}$ com primeiro mês após o tratamento cirúrgico e para $95 \mathrm{mg} / \mathrm{dL}$, três meses após. Morínigo et al. ${ }^{15}$ relataram que a glicemia pré-operatória foi de $114 \mathrm{mg} / \mathrm{dL}$ e, após um mês e meio, a taxa atingiu valores médios de $94,8 \mathrm{mg} / \mathrm{dL}$. Neste estudo, a média da glicemia, antes da operação, era de $96,55 \mathrm{mg} / \mathrm{dL}$ $( \pm 22,61)$. No grupo onde a variável foi analisada um mês após, obteve-se redução de $100,75 \mathrm{mg} / \mathrm{dL}( \pm 26,05)$ para $89,28 \mathrm{mg} / \mathrm{dL}( \pm 20,64)$ na taxa de glicose, que significou em melhora de $9,75 \%$. No grupo onde a variável foi analisada quatro meses após a operação, obteve-se redução de $100,8 \mathrm{mg} / \mathrm{dL}( \pm 29,99)$ para $87,4 \mathrm{mg} / \mathrm{dL}( \pm 10,5)$ na taxa de glicose, que significou em melhora de 10,34\%. Essa normalização da glicemia é de grande importância, visto que de acordo com trabalho realizado por Eberly et al. ${ }^{6}$, $44,3 \%$ dos casos de hiperglicemia acompanhados ambulatorialmente apresentam a síndrome metabólica.

$\mathrm{O}$ risco de mortalidade para pacientes que apresentam a síndrome metabólica tem sido estudado recentemente, havendo uma prevalência de $84 \%$ da síndrome nos casos de alto risco cardiovascular, principalmente quando associado a um grande número de fatores de risco para a SM. Eberly et al. ${ }^{6}$, que acompanharam pacientes por 18,4 anos, correlacionaram uma taxa de 3,623 mortes para cada 10 pessoas-ano, onde foram levados em consideração doença cardiovascular, doença arterial coronariana e a idade ajustada, e relatou que os eventos foram maiores nos pacientes portadores da síndrome metabólica do que nos que não a apresentavam $(\mathrm{p}<0,0001)$. A clara evidência entre a prevalência de dislipidemia e risco de mortalidade foi demonstrado, haja vista que em $88,9 \%$ dos pacientes com hipertrigliceridemia, em $68 \%$ dos que apresentavam altos índices de LDL e em $82,5 \%$ dos que apresentavam baixos índices de HDL, também havia o diagnóstico de síndrome metabólica ${ }^{2,18}$.

$\mathrm{O}$ aumento do tecido adiposo está ligado à resistência insulínica, provavelmente pelo efeito de diversas substâncias secretadas, dentre as quais os ácidos graxos livres, 
citocinas, a leptina, o Fator de Necrose Tumoral- $\alpha(\mathrm{TNF} \alpha)$ e a resistina, além da existência de fenômenos oxidativos citotóxicos (como a peroxidação lipídica), que contribuem para injúria mitocondrial e dano ao DNA. A obesidade provoca alterações no metabolismo da glicose em vários tecidos, o que promove hiperinsulinemia, apesar de também haver outros mecanismos reduzidos na reciclagem hepática de insulina. Em ratos, esses fatores alteram a ligação da insulina ao seu receptor e os fenômenos intracelulares pósreceptor, em sítios diversos como o fígado, os músculos e o próprio tecido adiposo. Isso ajuda a explicar a alta prevalência de diabete melito na obesidade mórbida (em torno de $20 \%)^{5,14,16}$.

Nessa pesquisa, ao analisar os lipidogramas dos pacientes acompanhados após um mês da operação, observou-se que o nível de colesterol total sofreu queda de $17,95 \%$ (com $\mathrm{P}<0,001$ ); o nível de HDL reduziu $14,05 \%$ (com $\mathrm{P}=0,005$ ); o nível de LDL sofreu queda de $15,31 \%$ (com $\mathrm{P}=0,016$ ); o nível de triglicerídeos sofreu redução de $30,29 \%$ (com $\mathrm{P}=0,002$ ). Já os pacientes acompanhados após quatro meses da operação, pôde-se verificar que o nível de colesterol total sofreu queda de $21,27 \%$ (com $\mathrm{P}<0,001$ ); o nível de HDL reduziu em 3,53\% (com $\mathrm{P}=0,126$ ); o nível de $\mathrm{LDL}$ reduziu em $23,72 \%$ (com $\mathrm{P}=0,016)$ e o nível de triglicerídeos sofreu queda de $29,6 \%$ (com $\mathrm{P}=0,007$ ).

Carvalho et al. ${ }^{4}$ encontraram redução dos níveis de triglicerídeos de $175 \mathrm{mg} / \mathrm{dL}$ para $145 \mathrm{mg} / \mathrm{dL}$ no $1^{\circ}$ mês de pós-operatório e para $120 \mathrm{mg} / \mathrm{dL}$ no $3^{\circ}$ e alteração mínima nos níveis de HDL (44 mg/dL no pré-operatório, $43 \mathrm{mg} /$ $\mathrm{dL}$ após 1 mês e $44 \mathrm{mg} / \mathrm{dL}$ no $3^{\circ}$ ). Morínigo et al. ${ }^{15}$ apresentaram redução na taxa de triglicerídeos de $138,1 \mathrm{mg} / \mathrm{dL}$ para $117 \mathrm{mg} / \mathrm{dL}$, decorridos um mês e meio da operação, e redução de HDL de 45,6 mg/dL para $42 \mathrm{mg} / \mathrm{dL}$ no mesmo período (com $\mathrm{P}<0,05$ ). Batsis et al.15 obtiveram colesterol total de $200 \mathrm{mg} / \mathrm{dL}( \pm 39)$, LDL de $118 \mathrm{mg} / \mathrm{dL}( \pm 33)$, HDL $45 \mathrm{mg} / \mathrm{dL}( \pm 11)$ e triglicerídeos de $190 \mathrm{mg} / \mathrm{dL}( \pm 119)$ com o tratamento cirúrgico, porém não foi estatisticamente significante quando comparado ao grupo de pacientes que receberam apenas tratamento clínico.

Kuk et al. ${ }^{10}$, relataram que os métodos para aferição do tecido adiposo visceral estão associados com alta incidência de síndrome metabólica $(\mathrm{P}<0,05)$, pois há evidências que sugerem que o mesmo ocorre pelo grande depósito de adipócitos viscerais metabolicamente ativos no omento e no mesentério. Esses autores utilizaram tanto os parâmetros do NCEP quanto do IDF e compararam pacientes com e sem o diagnóstico de síndrome metabólica, obtendo resultados estatisticamente significativos. Neste estudo os autores observaram que nenhum dos grupos submetidos à análise conseguiu êxito no alcance dos valores esperados como normais (a média das relações de cintura/quadril manteve-se superior a 0,9). No grupo acompanhado um mês após a operação houve redução de apenas $1,35 \%$, com $\mathrm{P}=0,137$, enquanto que no grupo acompanhado quatro meses, houve queda de $5,96 \%$, com $\mathrm{P}=0,02$.

\section{CONCLUSÃO}

Todos os parâmetros pré e pós-operatórios no $1^{\circ} \mathrm{e} 4^{\circ}$ mês tiveram melhora estatisticamente significante, com exceção do HDL-colesterol, após o bypass gastrointestinal em Y de Roux.

Nassif PAN, Lopes AD, Lopes GL, Martins PR, Pedri LE, Varaschim M, Bopp DS. Changes in the pre- and postoperative parameters in metabolic syndrome patients submitted to roux-en-y gastric bypass. ABCD Arq Bras Cir Dig 2009;22(3):165-70

ABSTRACT - Background - Overweight and obesity affects approximately 1.7 billion people, with increase in morbid obesity of $80 \%$ for women and $400 \%$ for men, since 1970. Metabolic syndrome is a complex disorder associated with insulin resistance and abdominal obesity, causing changes in glucose metabolism in various tissues and hyperinsulinemia. Aim - To verify changes in the pre and post-operative parameters in morbidly obese patients with metabolic syndrome who underwent to Roux-en-Y gastrointestinal bypass. Method - Seventy four patients with obesity with metabolic syndrome submitted to gastrointestinal bypass Roux-en-Y were included. All of them had measured waist-hip ratio, blood pressure, total cholesterol, HDL, LDL, triglycerides and fasting glucose pre-operatively and at the first and fourth month post-operatively. Results - In the first post-operative month, happened a weight reduction of 7.96\%, BMI of $8 \%$, waist-hip ratio of $1.35 \%$, total cholesterol of $17.95 \%$, HDL of $14.5 \%$, LDL of $15.31 \%$, TAG's of $30.29 \%$ and $9.75 \%$ of glucose; on the fourth month was observed a weight reduction of $14.94 \%$, BMI of $20.08 \%$, waist-hip ratio of $5.96 \%$, total cholesterol of $21.27 \%$, HDL of $3.53 \%$, LDL of $23.72 \%$, TAG's of $29.6 \%$ and $10.34 \%$ of glucose. Conclusion-All the parameters evaluated in this study had a statistic significant improvement, with the exception of HDL-cholesterol.

HEADINGS - Metabolic syndrome. Obesity. Gastrointestinal Roux-en-Y bypass.

\section{REFERÊNCIAS}

1. Alamo MA, Torres CS, Perez LZ. Vertical Isolated Gastroplasty with Gastroenteral Bypass: Preliminary Results. Obesity Surgery 2006; 16: 353-358

2. Batsis JA, Romero-Corral A, Collazo-Clavell ML, Saar MG, Somers VK, Lopez-Jimenez F. Effect of bariatric surgery on the metabolic syndrome : a population-based, long-term controlled study. Mayo Clinic 2008 Agosto; 83 (8): 897-906

3. Buchwald H, Avidor Y, Braunwad E, Jensen MD, Pories W, Fahrbach K. Bariatric surgery. A systematic review and meta-analysis. JAMA 2004; 292:1724-37

4. Carvalho PS, Moreira CLCB, Barelli MC, Oliveira FH, Guzzo MF, Miguel GPS. Cirurgia bariátrica cura Síndrome Metabólica? Arq Bras Endocrinol Metab 2007 Fev; 51(1)
5. Diniz MFHS, Diniz MTC, Sanches SRA, Rocha ALS, Ferreira JT, Sanches MD. Diabetes mellitus e cirurgia bariátrica: efeito da redução do peso no controle glicêmico. Rev Med Minas Gerais 2004;14(1):21-5

6. Eberly LE, Prineas R, Cohen JD, Vazquez G, Zhi X, Neaton JD, Kuller LH. Risk factor distribution and 18-year mortality in the multiple risk factor intervention trial. Diabetes Care Janeiro 2006; 29 (1) : 123-128

7. Francisco MC, Barella SM, Abud TG, Vilar VS, Reibscheid S, Arasaki CH. Análise radiológica das alterações gastrintestinais após cirurgia de FobiCapella. Radiol Bras 2007; 40(4): 235-238

8. Freitas ACT, Freitas DT, Parolin MB, Campos CAL, Coelho JCU. Doença Hepática Não-Alcoólica: evolução após derivação gastrojejunal em Y-de-Roux pela técnica de Fobi-Capella. Arq Gastroenterol 2007 jan/mar; 44(1): 49-53 
9. Gelonezes B, Pareja JC. Cirurgia Bariátrica Cura a Síndrome Metabólica? Arq Bras Endocrinol Metab 2006 Abril; 50 (2): 400-407

10. Kuk JL, Church TS, Blair S M, Ross R. Does measurement site for visceral and abdominal subcutaneous adipose tissue alter associations with the metabolic syndrome? Diabetes Care 2006 Março; 29 (3): 679-684.

11. Martins MVD, Souza AAP. Mecanismos cirúrgicos de controle do diabetes mellitus tipo 2 após cirurgia bariátrica. Rev Col Bras Cir 2007 Set-Out; 34 (5): 343-346

12. Martins MVDC. Porque o "by-pass" gástrico em y de roux é atualmente a melhor cirurgia para tratamento da obesidade. Rev bras videocir 2005;3(2):102104.

13. Mason EE. The mechanisms of Surgical Treatment of Type 2 Diabetes. Obesity Surgery 2005; 15: 459-461

14. Mattar SG, Velcu LM, Rabinovitz M, Demetris AJ, Krasinskas AM, BarinasMitchell E, Eid GM, Ramanathan R, Taylor DS, Schauer PR. Surgically-induced weight loss sigificantly improves nonalcoholic fatty liver disease and the metabolic syndrome. Annal os Surgery 2005 Out; 242 (4): 610-617
15. Morínigo R, Casamitjana R, Delgado S, Lacy A, Deulofeu R, Conget I, Barceló-Batllori S, Gomis R, Vidal J. Insulin resistance, inflammation, and the metabolic syndrome following Roux-em-Y gastric bypass surgery in severely obese subjects. Diabetes Care 2007 Julho; 30 (7): 1906-1908

16. Ramos APP, Abreu MRA, Vendramini RC, Brunetti IL, Pepato MT. Decrease in Circulating Glucose, Insulin and Leptin Levels and Improvement in Insulin Resistance at 1 and 3 months after Gastric Bypass. Obesity Surgery 2006; 16: 1359-1364

17. Santos R, Nunes A, Ribeiro JC, Santos P, Duarte JAR, Mota J. Obesidade, síndrome metabólica e atividade física: estudo exploratório realizado com adultos de ambos os sexos, da Ilha de S. Miguel, Região Autônoma dos Açores, Portugal. Rev. bras. Educ. Fís. Esp. 2005 out./dez; 19 (4): 317-28

18. Wong J, Molyneaux L, Constantino MI, Twigg SM, Yue DK. The metabolic syndrome in type 2 diabetes: When does it matter? Diabetes, Obesity and Metabolism 2006 Agosto; 8 : 690-697

Fonte de financiamento: não há Conflito de interesse: não há Recebido para publicação: 23/02/2009 Aceito para publicação: 28/04/2009 